\title{
Energy-Efficient Map Interpolation for Sensor Fields Using Kriging
}

\author{
Brian Harrington, Yan Huang, Member, IEEE, \\ Jue Yang, Student Member, IEEE, and Xinrong Li, Member, IEEE
}

\begin{abstract}
We propose a spatial-autocorrelation-aware, energy-efficient, and error-bounded framework for interpolating maps from sensor fields. Specifically, we propose an iterative reporting framework that utilizes spatial interpolation models to reduce communication costs and enforce error control. The framework employs a simple and low-overhead in-network coordination among sensors for selecting reporting sensors so that the coordination overhead does not eclipse the communication savings. Due to the probabilistic nature of the first-round reporting, the framework is less sensitive to sensor failures and guarantees an error bound for all functional sensors for each epoch. We then propose a graceful integration of temporal data suppression models with our framework. This allows an adaptive utilization of spatial or temporal autocorrelation based on whichever is stronger in different regions of the sensor field. We conducted extensive experiments using data from a real-world sensor network deployment and a large Asian temperature data set to show that the proposed framework significantly reduces messaging costs and is more resilient to sensor failures. We also implemented our proposed algorithms on a sensor network of MICAz motes. The results show that our algorithms save significant energy and the out-of-bound errors due to packet loss are below 5 percent.
\end{abstract}

Index Terms-Spatial autocorrelation, geosensor networks, sensor networks, energy efficient.

\section{INTRODUCTION}

SENSOR networks are expected to form a digital nervous System embedded in physical spaces and extend human beings" "tactile" sensations to every corner of the world. In recent years, coin- to palm-sized and programmable sensors have begun to be able to locate their positions, self-organize into a network, and communicate through multihop protocols with a gateway that incorporates longhaul communication capacity. This enables the deployments of robust distributed networks of hundreds to thousands of sensors.

In an acquisitional sensor network database [4], [38], [26], a collection of sensors of the same type may be treated as a table, e.g., lightSensors, in a database. The rows of the table are distributed among sensors in a physical space. Each sensor generates records in the format of <location, sensorID, reading,time $>$. Users can interact with the network using a declarative database query language. For example, users can issue queries such as "SELECT avg(readings) FROM lightSensors WHERE location IN P EVERY 5 seconds," where $P$ is a given polygon.

However, sensors are commonly resource constrained. Energy budgeting is particularly needed for long-term and remote sensor network deployments. Full duty cycle

- B. Harrington is with Yahoo!. E-mail: brh@yahoo-inc.com.

- Y. Huang is with the Department of Computer Science and Engineering, University of North Texas, 1155 Union Circle \#311366, Denton, TX 76203-5017. E-mail: huangyan@unt.edu.

- J. Yang and X. Li are with the Department of Electrical Engineering, University of North Texas, 1155 Union Circle \# 310470, Denton, TX 76203-5017. E-mail: \{jy0074, xinrong\}@unt.edu.

Manuscript received 18 June 2007; revised 31 Jan. 2008; accepted 21 Oct. 2008; published online 21 Nov. 2008.

For information on obtaining reprints of this article, please send e-mail to: tmc@computer.org, and reference IEEECS Log Number TMC-2007-06-0173. Digital Object Identifier no. 10.1109/TMC.2008.167.

$1536-1233 / 09 / \$ 25.00$ C 2009 IEEE operations on a sensor node, e.g., Berkeley Mica Motes, will deplete its energy supply in a few days. In-network aggregation [11], [32] has been proposed as one of the main mechanisms to reduce messaging cost due to the fact that communication is much more expensive than computation in sensors. However, the average/sum/max readings of a spatial region are often of limited use to domain scientists. Many phenomena in natural science, e.g., temperature, hydraulic head, and soil moisture, are actually continuous and, thus, best represented as continuous surfaces over the sensor fields. In fact, a raster surface map, e.g., soil moisture map, is frequently created by domain scientists using interpolation upon receiving readings from sensors and is fundamental for many subsequent field-based operations.

In this paper, we address the problem of interpolating maps from sensor fields. The naive way to interpolate the continuous spatial phenomena at the sink is to have all sensors report their readings and perform an interpolation at the sink. This approach depletes the energy of the sensor network very quickly. An alternative way is to utilize spatial autocorrelation to select a subset of sensors to report and then use them to interpolate a map at the sink. However, for the sensors that do not report, the estimation should be under a user-given error bound. Error-bounded data collection is sufficient, especially considering that sensors have calibration errors and residual errors are generally considered common for map interpolation. This problem is also referred to as the "SELECT*" problem with error bound in recent research publications [5].

One way to utilize spatial autocorrelation is to divide the sensors into groups and let the group leader aggregate/select the readings to report in the group and represent the whole group. Unfortunately, the group leader selection process is nontrivial and usually incurs 
substantial messaging cost if done dynamically in the sensor field. In some cases, the benefit of grouping cannot offset the overhead of dynamic group leader selection. On the other hand, static grouping may not be able to adapt to the dynamic topological changes in the field and frequent sensor failures.

In this paper, we propose a spatial-autocorrelationaware, energy-efficient, and yet error-bounded framework for interpolating maps from sensor fields that brings together the following innovations:

- A novel iterative reporting framework. The framework utilizes a simple probabilistic selection process to determine the sensors that need to report in the first round and relies on the other rounds to control reporting errors for all other sensors. The error bound is achieved by pushing spatial interpolation models used by the sink to sensors in the field, allowing the sensors to make decisions on the importance of their readings. The model utilizes spatial autocorrelation models, i.e., variograms, to allow a simple, localized, and energy-efficient innetwork coordination scheme among sensors so that the coordination overhead does not eclipse the communication savings. In addition, our framework can also increase the smoothness of the interpolated sensor field by increasing the number of reporting iterations. Smoothness of interpolation is an important requirement in many domains and has not been addressed by current sensor network literature.

- More resilient to sensor failures. The probabilistic nature of the first round allows the proposed model to adaptively select sensors that function normally at the beginning of each epoch. And reporting in the subsequent rounds is adaptive enough to rely on only reporting sensors in the previous rounds. As a result, the proposed model can always guarantee the error bound for the functional sensors in each epoch and interpolate the readings for a nonfunctional sensor using the error-bounded readings from the functional sensors (which is the best we can do for nonfunctional sensors).

- Graceful integration with temporal models. We propose a way to integrate any temporal data suppression model with our proposed framework. This allows an adaptive utilization of spatial or temporal autocorrelation, whichever is stronger in different regions of the sensor field.

- Extensive experimental evaluation and implementation of the proposed algorithms on MICAz motes. We performed extensive experiments using two data sets. One is from a real-world sensor network deployment from the Intel Berkeley Research Laboratory [24]. This data set is small with only 54 sensors. To further evaluate our framework, we evaluated various schemes using a large data set consisting of thousands of points for 600 months. We compare the proposed model with seven other models. Our experimental results show that the proposed model provides significant savings and is less sensitive to sensor failures.
We also implemented our proposed algorithms on a MICAz mote sensor network. The results show that our algorithms save significant communication costs and the out-of-bound errors due to package loss are below 5 percent.

The proposed approach will work well on long-term monitoring of smoothly varying fields (e.g., temperature) with strong spatial autocorrelation. It will capture "outlier" readings on largely smooth surfaces without consuming much more energy. However, this approach will not be applicable to detecting small-scale, sharp, and constant changes, e.g., chemical spills.

The rest of the paper is organized as follows: We discuss related work in Section 2. In Section 3, we first formally define the problem and present an overview of our framework. Then, we look into the details of using our framework with kriging as the spatial interpolation method along with a short discussion of other interpolation schemes. A method for integrating temporal schemes with our framework is presented in Section 4, followed by an extensive experimental study in Section 5. The paper is concluded with a discussion of possible future extensions of this work in Section 6.

\section{Related Work}

We classify related work in the broad area of sensor network databases into four categories: in-network aggregation, correlation-based sensor reporting, data compression, and interrogation.

Directed fusion [16] is a data-centric approach focusing on a general mechanism for query distribution and data collection. TinyDB [26] is a sensor network database system with a traditional SQL-like interface. Due to the resourceand communication-constrained nature of current sensors, query optimization schemes to reduce energy consumption are the focus of much research effort [27], [6], [25], [33], [32]. In particular, in-network aggregation is considered an effective way to reduce the messaging cost for aggregation queries (e.g., sum and average) at the cost of simple innetwork computations. The rationale is that communication is much more expensive than computation. In the TAG system [25], an aggregation tree is created when a query is broadcast to the sensors. The tree is used to aggregate the sensor readings from children to a parent all the way up to the root where the query originates. For distributive (e.g., sum, min, and max) and algebraic (e.g., average) aggregations, the TAG method significantly reduces the messaging cost by reducing message hops. With aggregation queries, detailed locational information is lost. Our work focuses on faithfully representing a field while reducing communication cost.

The other stream of work under the in-network aggregation category focuses on redistributing or aggregating sensor readings to designated in-network storage nodes to facilitate future queries [23], [12]. In-network storage does not reduce the number of sensors to report in order to create a map. Our work does not assume in-network storage but may be extended to include it. 
Correlation-based sensor reporting techniques utilize spatial and/or temporal correlation. Traditional temporal suppression schemes from stream processing that utilize approximate caching [29], time-series models [22], and Kalman Filters [17] have been adapted for mote-sized sensors. Approximate caching [29] relies on cached values to reduce the number of reports needed from the data stream to the sink. More sophisticated time-series models to capture the temporal trends of the sensor readings have been used in [22] and [13]. Each sensor node calculates a function based on past readings to predict the readings of the node in the near future and sends it to the sink. In the case of high temporal autocorrelation, a time series is condensed into a single function, thus reducing communication cost. Our work focuses on utilizing spatial autocorrelations and is orthogonal to models that use temporal autocorrelation. Temporal models can be integrated with our framework, which will be discussed in Section 4.

Utilizing spatial autocorrelation has been suggested in prior research work. The clustering-based approaches [13], [2], [34], [35], [14] group sensor nodes according to the spectrum of sensing values or spatial proximity and then select leaders to represent the group. Election and voting algorithms are important in selecting the representatives for a group of value-correlated sensors [13], [40]. These algorithms must be distributed and localized in order to scale well for large sensor networks. Energy needs to be budgeted among representative election and communication of selected sensors. Snapshot [19] investigated various heuristics for electing a small set of representative nodes in the network in a localized manner to form a snapshot of the network and provide quick approximate answers to user queries. Unlike the scheme we propose in this paper, the representative selection process in Snapshot can only be performed very infrequently to achieve overall saving. In Ken [5], sensors are partitioned into disjoint cliques with one sensor in each clique selected as the leader of the clique. The leader assumes the duty of selecting a subset of data to be sent back to the sink according to a dynamic probabilistic predication model. The dynamic probabilistic predication model is obtained by a set of training data and is maintained by both the sink and the sensor field. Thus, the sink can calculate the expected readings for sensors that do not report using the same predication model as the sensor field. Compared to Ken, our model has a simple probabilistic voting process to select sensors to report in the first round and relies on spatial interpolation models to control errors in the other rounds, which can be more resilient to sensor failures.

Data compression may be performed spatially or temporally in sensor networks. The information-theoretical approaches [8] aim to find an optimal rate to compress redundant information in individual sensor readings. The joint routing and source coding approach [20], [30], [31] attempts to compress redundant information along the routing paths to reduce the number of bits transmitted. For these techniques, the number of transmitted messages are condensed but not reduced. Data compression is orthogonal to and may be applied on top of correlation-based data suppression.

Selectively interrogating sensors is another way to avoid requiring all sensors to report. Bash et al. proposed an energy-efficient and uniform sampling scheme for sensor networks [3]. A random sampler from the central station probes the sensor network to select the set of samples. A sensor uses its Voronoi cell to decide a probability to accept or reject the probe. Uniform sampling is useful for application domains such as querying the average sensor battery life. Other approaches include the Binocular system [10]. This approach divides sensors into working and sleeping sets and only collects data from the working set. A system model is used to estimate the values for the sleeping set. To avoid error accumulation the system model is updated by having all sensors report at some specified interval. Recently, Deshpande et al. [9] has proposed a model-based probabilistic approach in the central site to answer queries, which samples a few sensors when necessary to improve the estimation and achieve a confidence-level guarantee. In general, the interrogation-based approach is "pull based" (compared with a "push-based approach" such as Ken [5] and our model). A "pull-based approach" does not provide an error guarantee and is insensitive to outliers, which are important for application domains such as environmental monitoring.

\section{E2K FRAMEWORK}

In this section, we will provide a formal definition of the problem and present our proposed Error-Bounded EnergyEfficient Kriging (E2K) framework. In addition, we discuss how to select an appropriate spatial interpolation model, which is very important in our framework.

Problem statement. Let $S$ be a set of spatially distributed sensors that monitor some attribute $A$ at a time instant $t \in T$, and for $s \in S$, let $Z_{t}(s)$ be the value of $A$ for sensor $s$ at time $t$. Let $C$ be a central collection sink that processes the information received from $S$ and let $Z_{t}^{*}(s)$ be the value $C$ estimates for $A$ for sensor $s$ at time $t$. Devise an algorithm for $C$ and the sensors in $S$ such that for all $s$ and $t$, the estimated value is within a user-specified error threshold $\epsilon>0$ from the actual value, i.e., $\left|Z_{t}(s)-Z_{t}^{*}(s)\right|<\epsilon$, with the objective of reducing the total messaging cost and the constraint of interpolating a map as smooth as possible.

Reducing the total messaging cost is chosen as the objective because sending and receiving messages dominate the energy consumption in current sensor networks [5], [9], [25]. When a message is sent from a sensor, all the neighbors of that sensor will receive the message even though in many cases only a subset of the neighbors are intended destinations. Because sending and receiving have similar energy cost, it is fair to use the total message count sent and relayed from all sensors as the message cost (the actual total message cost that includes receiving and sending messages will be some multiple of the total message cost that we use). From the perspective of natural science communities, a smoothly interpolated map is very important.

Tobler's first law of geography [7] states that in space, everything is related to everything else, but nearby things 


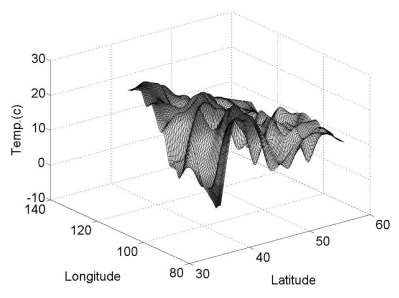

(a)

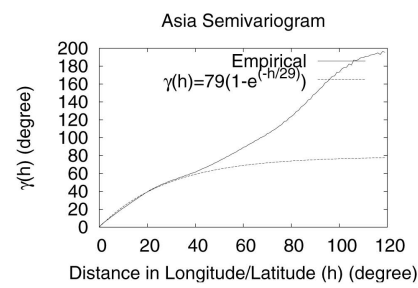

(b)
Fig. 1. Sample spatial autocorrelation and empirical and theoretical variograms.

are more related than distant things. For example, Fig. 1a shows temperatures from part of Asia in June 1951 [1] (this data set will be described further in Section 5). The temperature surface is smooth and shows spatial autocorrelation, where close-by locations have closer readings than locations that are far away from each other. Various models, e.g., variograms, Moran's I, and Geary's C [7], have been developed to describe this phenomena (formally spatial autocorrelation) and have been incorporated into spatial interpolation models to create maps. Our work is mainly inspired by spatial interpolation models utilizing spatial autocorrelation. First, we address the problem of utilizing spatial autocorrelation for reducing the messaging cost. Then, we address the problem of answering longrunning queries that can utilize temporal autocorrelation in Section 4.

\subsection{The Iterative Reporting Framework}

As shown in Fig. 2, map queries are injected from a user interface into the sensor field. Each sensor makes a local decision about the importance of its reading and decides if it needs to report. As a result, only a subset of the sensors (red in color or gray in black-and-white prints in Fig. 2) will actually report. The reporting sensors route their readings through any multihop protocol [39], [18], e.g., GPSR [18], to the gateway sensor or sink. The sink estimates the values for nonreporting sensors and then interpolates a raster map from the sensor values using the same spatial interpolation model that was used in the field.

The key challenge here is the trade-off between the complexity of coordination among sensors in dynamically deciding which ones should report and the savings that result from having fewer sensors report while maintaining an error bound for all sensors. We need to make sure that the coordination overhead does not eclipse the communication savings. The three main (often-conflicting) goals are 1) minimizing the number of sensors that report and thus saving energy, 2) minimizing coordination costs among sensors, and 3) allowing the sink to interpolate readings for nonreporting sensors within an error threshold $\epsilon$.

As stated in the related work, the Ken framework [5] employed a disjoint-group-lead-by-a-leader mechanism to allow group leaders to select a subset of sensors to report inside the group and use an agreed upon probabilistic model by the sink and sensor field to interpolate the readings for nonreporting sensors. We explore an alternative approach where all sensors are peers without

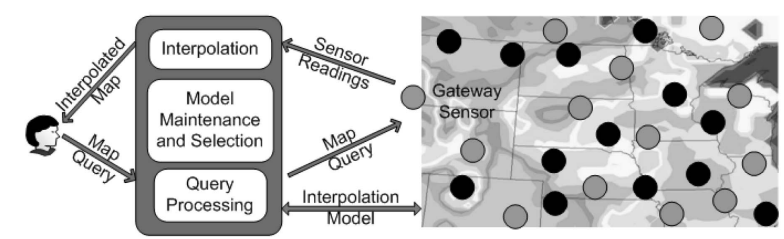

Fig. 2. System overview.

designated leaders, which may lead to better resilience to sensor failures.

A naive probabilistic approach would let each sensor report with a probability $p$. This approach does not require coordination. However, there is no error bound for sensors that do not report. An alternative would be to have all sensors send their value to neighbors. Each sensor interpolates its own reading using the readings from its neighbors. If the interpolated value deviates from the real reading by more than $\epsilon$, then the sensor reports. This approach again does not have an error guarantee for nonreporting sensors because of concurrent decision making and error propagation in sensor fields. We propose an iterative reporting framework called $\mathrm{E} 2 \mathrm{~K}$ that is both energy efficient and has an error guarantee. E2K consists of Algorithm 1 for individual sensors and Algorithm 2 for the central site.

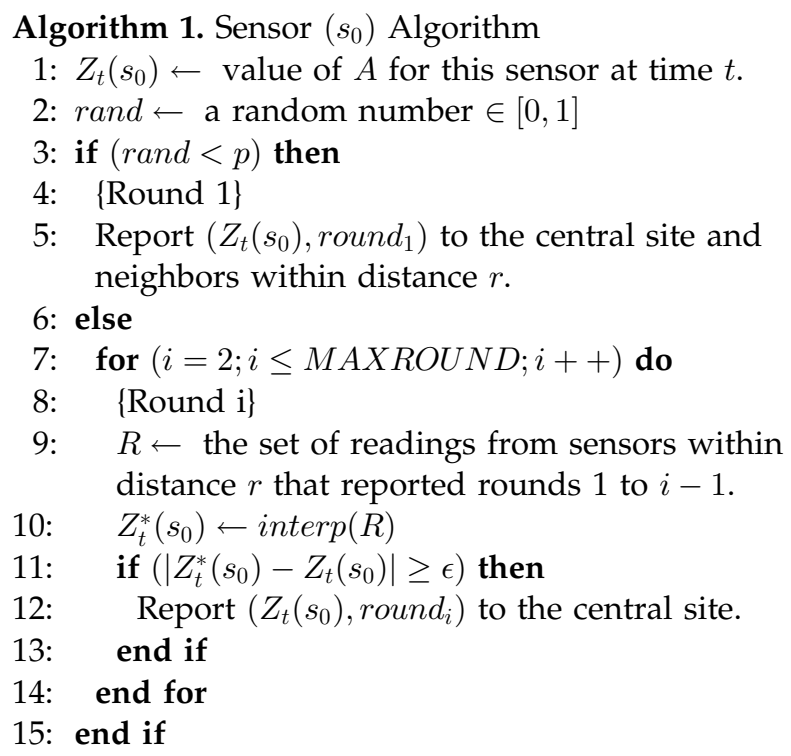

The algorithm for sensors is divided into several rounds. In the first round, a sensor decides to report with a probability $p$. As a result, a set of representative sensors are selected to temporarily represent the field. Reporting sensors route their readings to the sink and also send their readings to all sensors within distance $r$ for use in the later rounds. In the following rounds, a sensor not yet reported will interpolate its reading using the readings received from reporting sensors so far. If the estimated value deviates from the real value by more than $\epsilon$, then it reports. The process continues until the $M A X R O U N D$ is reached or there is nothing to report in a round. The last round is needed because the previous rounds do not have error bounds for nonreporting sensors. 
Any kind of interpolation method can be used in the later rounds, e.g., simple average. However, a better interpolation method results in fewer sensors that need to report. Basically, the probabilistic first round provides a reasonable number of sensors to report so that the estimation methods will work well in the later rounds, and the better the interpolation method is the better the overall result will be. We will revisit spatial interpolation methods in Section 3.2.

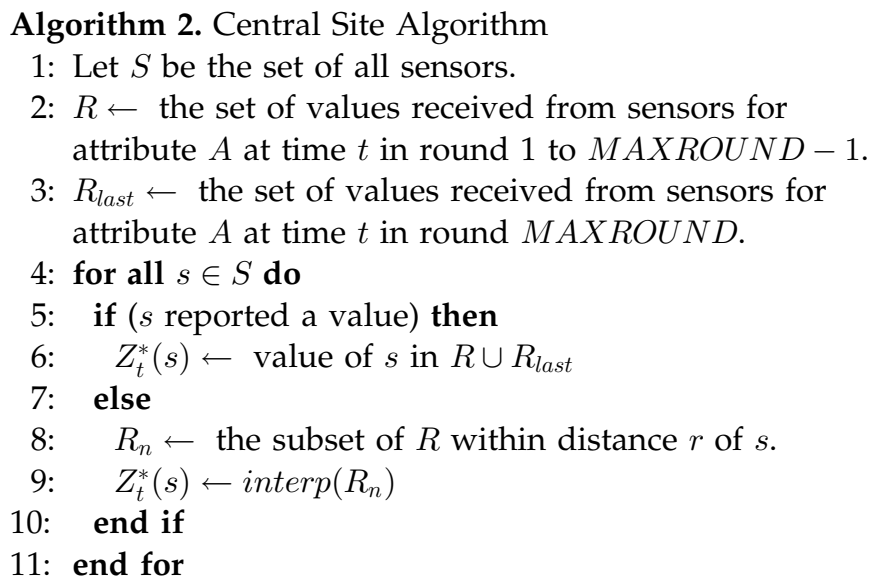

The algorithm for the central site is meant to mirror what is done by the individual sensors. If a reading is sent by a sensor, then that reading will be used. For sensors where no reading was received, then the reading will be estimated using the same method and the same set of neighbors that the sensor used to determine whether to report in the later rounds, which gives us an error bound for each sensor location of $\epsilon$. More formally, we have the following lemma:

Lemma 1 (error bounding). Assuming an ideal sensor network, i.e., no sensor failure and no package loss, for any sensor s, let $Z_{t}(s)$ be the actual value and $Z_{t}^{*}(s)$ be the estimated value of $s$ for attribute $A$ at time $t$. Then, $\left|Z_{t}^{*}(s)-Z_{t}(s)\right|<\epsilon$ using the proposed algorithms for each sensor and the central site.

Proof. There are two cases to consider:

1. If $s$ reported its value, then $Z_{t}^{*}(s)=Z_{t}(s)$, which implies that $\left|Z_{t}^{*}(s)-Z_{t}(s)\right|=0<\epsilon$ since from the problem statement $\epsilon>0$.

2. If $s$ did not report its value, then $Z_{t}^{*}(s)=\operatorname{interp}\left(R_{n}\right)$, where $R_{n}$ is the set of values that reported in all the rounds except the last round and that are within distance $r$ from $s$. This is the same as the estimated value used in the last round for sensor $s$. If $\left|Z_{t}^{*}(s)-Z_{t}(s)\right| \geq \epsilon$, then $s$ would have reported. Therefore, $\left|Z_{t}^{*}(s)-Z_{t}(s)\right|<\epsilon$.

If the objective is to have error bounding, then setting $M A X R O U N D=2$ will be sufficient. However, it may result in a partially smooth map based on the first-round reported values and interpolated values using the first-round values, punctuated by discontinuities by the second-round reported values. If providing a continuous map is also an objective, then increasing the value of $M A X R O U N D$ so that the punctuation by sensors reporting in the last round is

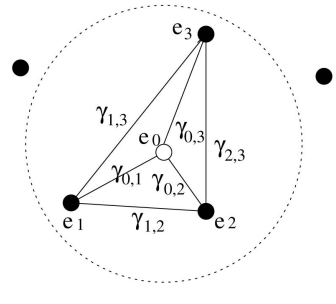

Fig. 3. Kriging example.

minimized or eliminated would be useful. Thus, the iterative approach not only achieves the primary goal of bounding errors but also results in the additional benefit of providing a continuous interpolated map.

\subsection{E2K: Choosing Interpolation Method}

Although our framework is general with respect to the interpolation method used and always guarantees an error bound, the interpolation method used has a great impact on the performance of the E2K framework. Furthermore, we need to make sure that the sensor field can handle sophisticated interpolation models without incurring too much storage and computation cost.

Let $Z(e)$ be a random function at a location $e$ of a sensor field. The value of a location $e_{0}$ is estimated by a linear estimator using the neighbors, $e_{1}, e_{2}, \ldots, e_{n}$, within distance $r$ :

$$
Z^{*}\left(e_{0}\right)=\sum_{i=1}^{n} \lambda_{i} Z\left(e_{i}\right),
$$

where $Z^{*}\left(e_{0}\right)$ represents the estimated value at location $e_{0}$, and $\lambda_{i}$ refers to the weight given to the $i$ th neighbor's value. For example, in Fig. 3 , we wish to estimate a value at $e_{0}$, using the data values from the $n$ neighboring sample points $e_{i}$, $i=1,2,3$. So we have $Z^{*}\left(e_{0}\right)=\lambda_{1} Z\left(e_{1}\right)+\lambda_{2} Z\left(e_{2}\right)+\lambda_{3} Z\left(e_{3}\right)$.

There are a number of ways to assign the weights. One way would be to assign equal weights to all neighbors, i.e., to take a simple average of the neighbor values (Simple Average). Another scheme would be to use the inverse of the distance as the weights, i.e., $\lambda_{j}=\frac{1 / d_{j}}{\sum_{i=1}^{n} 1 / d_{i}}$, where $d_{i}$ is the distance from $e_{i}$ to $e_{0}$ (Inverse Distance). However, these techniques do not examine the spatial structure of the data and may result in large estimation errors and, thus, large communication cost.

Kriging [7], [36] is widely used and has a long history of popularity in many natural science domains. It is a best fit linear unbiased estimator of a spatial variable at a particular site or geographic area. The goal of kriging is to create a raster map of a given resolution to represent a surface using a set of sample readings. It estimates a value at a location of a region for which a covariance/variogram is known or can be estimated using data in the neighborhood of the estimation location.

We assume that $Z(e)$ is second-order stationary. This means that the expected value $E[Z(e)]=m$, where $m$ is the mean, for any point of the domain, and the covariance between any 
pair of locations depends only on the vector $h$ that separates them, i.e., $C(h)=C(e, e+h)=E[Z(e) \times Z(e+h)]-m^{2}$. We chose ordinary kriging due to its adaptivity to local conditions, i.e., it only requires local second-order stationarity as opposed to global.

Kriging assigns weights according to a known or estimated covariance/variogram function that captures the spatial autocorrelation. The variogram $2 \gamma_{z}(h)$ is defined as $\operatorname{Var}[Z(e+h)-Z(e)]$. Using sampled data, the semivariogram is estimated as

$$
\hat{\gamma}_{z}(h)=\frac{1}{2 N_{h}} \sum_{i=1}^{N_{h}}\left(z\left(e_{i}\right)-z\left(e_{i}+h\right)\right)^{2},
$$

where $N_{h}$ is the number of pairs of samples whose distance from each other is $h$.

The empirical semivariogram is then fit with a theoretical model. For example, Fig. 1b shows the empirical semivariogram for an Asia temperature data set [1] fit with the exponential model $\gamma(h)=79\left(1-e^{(-h / 29)}\right)$. Please note that in a wireless sensor network, interpolation using sensor values too far away will incur substantial communication costs (energy consumption). Thus, we tried to match the empirical and theoretical variograms as much as possible for small distances.

There are two important parameters of the variogram model: the range and the sill. The sill is an asymptotic bound the variogram reaches. The range is the distance it takes for the variogram to reach the sill. In practice, range is the value of $h$ when $\gamma(h)$ reaches 95 percent of the sill. In Fig. 1b, the sill is 79 , and the range is $-\ln (1-95$ percent $) \times 29=86.88$. The covariance can be expressed in terms of the variogram as $C(h)=C(0)-\gamma(h)$.

Ordinary kriging is unbiased, and the unbiasedness condition states that

$$
\sum_{i=1}^{n} \lambda_{i}=1
$$

The kriging variance is

$$
\sigma_{E}^{2}=C(0)-2 \sum_{i=1}^{n} \lambda_{i} C\left(e_{i}, e\right)+\sum_{i=1}^{n} \sum_{j=1}^{n} \lambda_{i} \lambda_{j} C\left(e_{i}, e_{j}\right) .
$$

Optimal values for weights $\lambda_{i}, i=1,2, \ldots, n$, are obtained by minimization of kriging variance through the standard method of taking first derivatives with respect to each weight $\lambda_{i}$ and setting them to zero. The Lagrange parameter $\mu$ is added in the minimization of the kriging variance to honor the unbiasedness condition of the ordinary kriging. The weights can then be estimated by the following system of equations:

$$
\left(\begin{array}{c}
\lambda_{1} \\
\vdots \\
\lambda_{n} \\
\mu
\end{array}\right)=\left(\begin{array}{cccc}
\gamma\left(e_{1}, e_{1}\right) & \ldots & \gamma\left(e_{1}, e_{n}\right) & 1 \\
\vdots & \ddots & \vdots & 1 \\
\gamma\left(e_{n}, e_{1}\right) & \ldots & \gamma\left(e_{n}, e_{n}\right) & 1 \\
1 & 1 & 1 & 0
\end{array}\right)^{-1}\left(\begin{array}{c}
\gamma\left(e_{1}, e_{0}\right) \\
\vdots \\
\gamma\left(e_{n}, e_{0}\right) \\
1
\end{array}\right)
$$

Because kriging is preceded by an analysis of the spatial structure of the data by using a variogram model to integrate the average variability into the estimation model, the interpolation is likely to be more accurate than simple models. For the ordinary kriging that we described, the interpolation is exact, meaning that if a sample value is available at the location of estimation, the kriging solution is equal to that value.

The two main issues in the E2K framework using kriging are training and processing. Unlike the simple average and inverse distance schemes, kriging uses a variogram that is determined using previous data. Fortunately, all the training could be performed at the sink where resources are not constrained. After the training, only a few values used to describe the theoretical variogram need to be disseminated to the sensor network. Specifically, we use a training surface to obtain an empirical variogram at the sink. Then, a theoretical variogram is fit to the empirical variogram with the goal of fitting the data with distance less than $r$ as close as possible, again at the sink. Currently, we select variograms and parameters manually. We chose the one that seems to fit many snapshots. Of course, the variograms will not be the best and are not supposed to stay constant in time. However, as our goal is to reduce the message cost while still guaranteeing an error bound as many other methods in the literature, the "bad" variograms only reduce our savings. Automating the variogram selection process will be interesting and useful, and adaptively changing variograms over time will help query optimization, which may be explored in the future. The theoretical variogram described by type (exponential, spherical, etc.) and associated parameters are provided to each sensor. As such, a fairly small amount of information needs to be sent to the sensors to allow the sensor field and the sink to have the same interpolation model.

In terms of processing, the main requirement is that the sensor is capable of solving the linear system once it determines which neighbors report each round. Due to the computation constraint of sensors, a large linear system is not desirable. Fortunately, for kriging, not many neighbors are needed to interpolate a value. E2K achieves a desired number of neighbors for each sensor by setting the value of the probability to report in the first round in our sensor-side algorithm to $\frac{n_{\text {desired }}}{n_{\text {current }}}$, where $n_{\text {desired }}$ is the number of neighboring sensors desired, and $n_{\text {current }}$ is the current number of neighboring sensors within the distance $r$. With a sensor deployment following a Poisson distribution, we expect $\frac{n_{\text {desired }}}{n_{\text {current }}}$ percent of all the sensors will report in the first round. By setting a small value for the $n_{\text {desired }}$ parameter, e.g., five, it also results in the beneficial effect of dynamically adapting to the density of the sensor network and provides more savings for dense sensor networks with a similar level of spatial autocorrelation.

The only messages that need to be sent for coordination are the messages sent by the sensors that decide to report in the each round to their neighbors. However, since the sensors that decide to report in each round need to send their readings to the sink, the other sensors in the neighborhood can eavesdrop the reading. So when the interpolation neighborhood is less than the radio range of the sensors, the coordination cost in terms of messaging is zero. Formally, we have: 
Lemma 2 (conditional zero coordination cost). In $E 2 K$, when the spatial interpolation neighborhood is less than or equal to the radio range, the number of messages sent in order to coordinate sensors in deciding which ones need to report and maintain an error bound is zero.

\subsection{Resilience to Sensor Failures}

In a real sensor network deployment, node and communication failures are very common, due to weather conditions, breakage, and low battery power. For node failures, similar to Ken [5], E2K depends on the expected reporting rate or periodical probing to distinguish failed sensors from nonreporting functional ones. Before a sensor failure is detected, the best a model can do is to interpolate its value of a failed sensor using readings from other sensors, although we could not say much about the accuracy of the interpolated value since we do not know its real value. However, for normal functioning sensors, we still want to have the error guarantee.

In a static grouping and leader designation scheme such as that employed by Ken, leaders are selected, and leader information is disseminated to individual sensors at the sensor network setup time or periodically. The cost is amortized when many queries or long-running queries use the leaders selected to save costs. During a long-running query, e.g., several hours, it is highly possible that some leaders will fail. Leader failure is usually more costly than nonleader failures. If a leader of a group fails, the readings from the whole group are lost. As a result, the sink will assume that the readings of the whole group can be interpolated. Although some of the sensors in the group could have failed as well and their readings need to be interpolated anyway, for the rest of the functional sensors in the group, their interpolated values at the sink may not have an error bound due to leader failures.

During each epoch of a long-running query, a sensor collects one sample of the data and transmit the data to the sink through a multihop protocol. The time it takes to sample and send a value varies for different sensor networks but is usually in the magnitude of milliseconds. For example, for MICAz, sampling and transmission takes around $4 \mathrm{~ms}$, and for MICA2, this could take up to $5 \mathrm{~ms}$. We say that a sensor field is epoch reliable if the set of failed sensors stays the same during the iterative sampling period (a few milliseconds) of the E2K framework for each epoch. Compared with the assumption that a sensor field is reliable during a long-running query (a few hours to a few months), epoch reliability is much more realistic. In a distributed and dynamic scheme such as E2K, representative sensors in the first round are dynamically decided through a probabilistic voting scheme for each epoch. In the following rounds, sensors make their reporting decisions based on reporting sensors in the previous rounds. As a result, as long as the sensor network is epoch reliable and guarantees packet delivery, E2K guarantees that the interpolated values for all functioning sensors are within the user-given error (the possible partitioning of the sensor network problem is not addressed and is out of scope of this paper).

Fig. 4 illustrates a static grouping scheme and the proposed E2K scheme when $M A X R O U N D=2$. Failed sensors are represented by $\bullet$, reporting sensors are

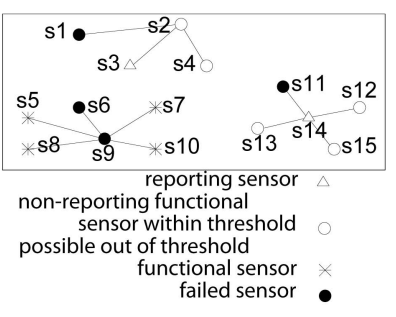

(a) (b)

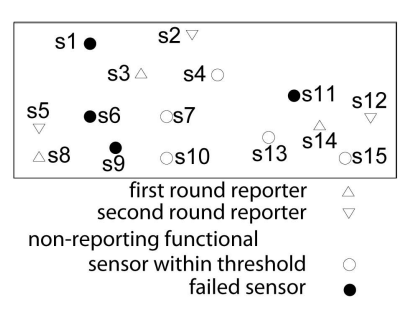

Fig. 4. Resilience to sensor failure. (a) Static grouping. (b) E2K.

represented by $\triangle$ (for E2K, sensors that report in the first round are represented by $\triangle$, and sensors that report in the second round are represented by $\nabla$ ), and nonreporting sensors that function normally during the current epoch with an error guarantee are represented by $\circ$. Note that for E2K, all nonreporting functional sensors have an error guarantee. For the static grouping scheme, $s_{9}, s_{2}$, and $s_{14}$ are static group leaders. When $s_{9}$ fails, although functional, $s_{5}$, $s_{6}, s_{7}$, and $s_{8}$ will not report, resulting in possible out-ofbound value interpolations at the sink.

Lemma 3 (error bounding for functional sensors). In an epoch-reliable and zero-packet-loss sensor field, for any normal functioning sensor $s$, let $Z_{t}(s)$ be the actual value and $Z_{t}^{*}(s)$ be the estimated value of $s$ for attribute $A$ at time $t$. Then, $\left|Z_{t}^{*}(s)-Z_{t}(s)\right|<\epsilon$ using the E2K framework.

We omit the proof of the lemma due to the space constraint. The proof is similar to that of Lemma 1 assuming that a new sensor field is formed by those normal functioning sensors.

For a sensor network with packet loss, which is more common, in order to distinguish packet loss from no report, we can use reliable unicast communication by retransmitting packets that are not acknowledged by the destined sensors if desired. We will experimentally show in Section 5.2 that the out-of-bound errors due to packet loss in our real MICAz sensor network are low.

\section{Temporal E2K}

As discussed before, with a sensor deployment following a Poisson distribution, we expect $\frac{n_{\text {desired }}}{n_{\text {current }}}$ percent of all the sensors will report in the first round. $|S| \times \frac{n_{\text {desired }}}{n_{\text {current }}}$ becomes the lower bound in terms of the total number of sensors that need to report for E2K and is obtained when no sensor needs to report in the second round.

Many times, sensor readings exhibit both spatial and temporal autocorrelation, especially for localized regions where temporal autocorrelation is so high that the temporal suppression alone can predict all of the values in the region well. Our proposed scheme takes advantage of spatial autocorrelation using interpolation models. However, sometimes, better results, e.g., having less than $|S| \times \frac{n_{\text {desired }}}{n_{\text {current }}}$ report, are achievable through temporal autocorrelation, especially when the sampling rate is very high, and thus, there is high temporal autocorrelation between subsequent readings. We propose an approach that can easily integrate any temporal models with E2K. This approach adaptively 


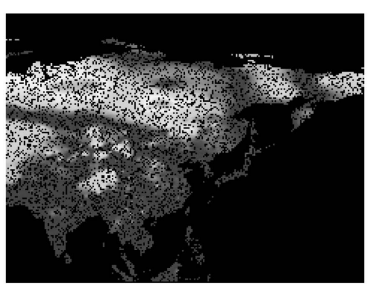

(a)

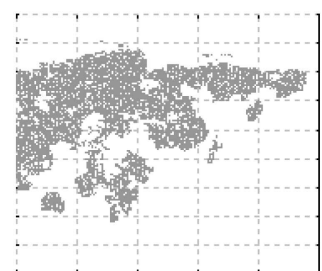

(b)

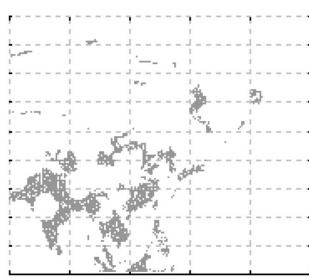

(c)

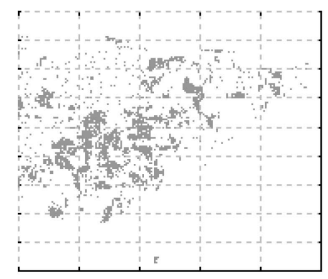

(d)

Fig. 5. Residual map and predication method map. (a) Residual map. (b) Nonreporting due to spatial. (c) Nonreporting due to temporal. (d) Reporting sensors.

utilizes spatial or temporal autocorrelation based on whichever is stronger in the local neighborhood of the sensor field.

The basic approach is summarized in Algorithm 3. In the first round, a sensor whose predicated value based on a temporal autocorrelation scheme differs from its real value by more than $\epsilon$ will report with probability $p$. In the later rounds, those sensors that have not reported yet and have more than $n_{\min }$ neighbors reported already will spatially interpolate its value, compare with the actual value, and report if the two values differ for more than $\epsilon$. The rest of the sensors that have not reported yet do not have enough reporting neighbors and will use the temporally predicated value to decide whether to report again. In this scheme, if a region has strong temporal correlation, none of the sensors for that region will report.

\section{Algorithm 3. Temporal Sensor $\left(s_{0}\right)$ Algorithm}

1: $Z_{d}\left(s_{0}\right) \leftarrow$ a temporal predicated value of $A$ for $s_{0}$ using previously reported readings of $s_{0}$.

2: $Z_{t}\left(s_{0}\right) \leftarrow$ value of $A$ for this sensor at time $t$.

3: $p \leftarrow \frac{n_{\text {desired }}}{n_{\text {current }}}$

4: rand $\longleftarrow$ a random number $\in[0,1]$

5: if $\left(\left|Z_{t}\left(s_{0}\right)-Z_{d}\left(s_{0}\right)\right| \geq \epsilon\right.$ and rand $\left.<p\right)$ then

6: $\{$ Round 1$\}$

7: $\quad$ Report $\left(Z_{t}\left(s_{0}\right)\right.$, round $\left._{1}\right)$ to central site and neighbors within distance $r$.

8: $\quad Z_{d}\left(s_{0}\right) \leftarrow Z_{t}\left(s_{0}\right)$

9: else

10: for $(i=2 ; i \leq M A X R O U N D ; i++)$ do

11: $\quad\{$ Round i

12: $\quad R \leftarrow$ the set of readings from sensors within distance $r$ that reported in the rounds 1 to $i-1$.

13: $\quad$ if $\left(\operatorname{size}(R)>n_{\min }\right)$ then

14: $\quad Z_{t}^{*}\left(s_{0}\right) \leftarrow \operatorname{interp}(R)$

15: $\quad$ if $\left(\left|Z_{t}^{*}\left(s_{0}\right)-Z_{t}\left(s_{0}\right)\right| \geq \epsilon\right)$ then

16: $\quad$ Report $\left(Z_{t}\left(s_{0}\right)\right.$, round $\left._{i}\right)$ to central site.

17: $\quad Z_{d}\left(s_{0}\right) \leftarrow Z_{t}\left(s_{0}\right)$

18:

19:

20:

21:

else

if $\left(\left|Z_{t}\left(s_{0}\right)-Z_{d}\left(s_{0}\right)\right| \geq \epsilon\right)$ then

Report $\left(Z_{t}\left(s_{0}\right)\right.$, round $\left.i\right)$ to central site.

$Z_{d}\left(s_{0}\right) \leftarrow Z_{t}\left(s_{0}\right)$

end if

end if

end for

end if
At the sink, for each temporal snapshot, sensor readings are recovered by the new modified method in order to mirror the behavior in the sensor field and bound the error. This is achieved by the following scheme:

- If a nonreporting sensor has less than $n_{\min }$ neighbors that report in rounds 1 to $M A X R O U N D-1$, then use the temporal prediction as the estimation of the sensor reading.

- $\quad$ Otherwise, use the neighbors that report in rounds 1 to $M A X R O U N D-1$ to spatially interpolate the value for the sensor.

The $n_{\min }$ parameter is an important quality control mechanism for spatial interpolation and a communication channel for the sink and field to agree upon the choice between spatial and temporal interpolation. In the integrated scheme, spatial and temporal models are "fighting" with each other to estimate a sensor's value. In general, spatial interpolation needs at least some number of neighbors, e.g., three, to interpolate well. When a sensor does not have enough neighbors that report in the previous rounds to spatially represent it, it is better for temporal suppression to give it a try.

Fig. 5a shows the residual map (subtracting the mean from the reading for each sensor) of the Asian temperature data with 75 percent sensor density in January 1960. From blue to yellow (dark to light shade in black and white print), the residuals increase. Fig. $5 b$ shows the sensors that decided not to report based on spatial interpolation. Fig. 5c shows nonreporting sensors due to temporal predication, and Fig. $5 \mathrm{~d}$ shows reporting sensors for the same data set. As can be seen, the temporal model helps to reduce a large number of sensors that need to report in South Asia near the equator where temperature is more consistent throughout the year and temporal autocorrelation is high.

\section{Evaluation}

The performance of E2K was evaluated using simulations and a real sensor network. The simulation was performed on two data sets:
1. Lab. This data set refers to an Intel lab data set [24] consisting of 54 sensors running for a little over a month, monitoring temperature, voltage, humidity, and light. This data contains traces from a real sensor network deployment including network failure information, so it is particularly useful for examining failures. 
2. Asia temperature. This data set refers to the Asian portion of a data set created using station records from the Global Historical Climatology Network (GHCN) and Legates and Willmott's [21] data sets for monthly precipitation and air temperature. We used version 1.02 of this data set, released in July 2001, which contains data for each month from January 1950 to December 1999 [1]. The Asia subset was sampled randomly to get two densities with 25 percent and 75 percent of the points, respectively. In our experiments, these sampled points were treated as sensors that formed a sensor network. This data set provides a large number of sensors in an outdoor environment where spatial correlations are stronger.

We implemented the algorithms on a sensor network of 25 MICAz motes [15]. Each MICAz mote [15] was equipped with a MTS310 sensor board [15]. The gateway sensor is installed on an extension interface board (MIB510) [15] through which the sensor can talk to a PC using serial communication. The motes were configured in a $5 \times 5$ grid with a length of $4 \mathrm{ft}$ from one mote to another. Each sensor measured the light intensity generated by a projector.

Because most algorithms in the literature only concern about the objective of error bounding, we set the $M A X R O U N D$ to two for the simulation experiments for fair comparison. In real sensor network implementation and experiments, we set the MAXROUND to eight in order to generate smooth maps.

\subsection{Simulation Experiments}

We compare our model with the state-of-the-art Ken model [5] along with six other simple models on the two data sets:

- A baseline model TinyDB (T) [26]. Every sensor will report in every epoch. The error is always zero; thus, it is bounded in this scheme.

- Three simple models where each sensor only reports if its readings deviate from a reading agreed upon by both the sensor field and the sink by more than $\epsilon$ :

1. Global average (G). Both the sink and the sensors keep a global average reading of all sensors obtained from the training data. If a sensor deviates from the global average by more than $\epsilon$, then it reports.

2. Approximate caching (A) [29]. Every sensor caches the last reported reading to the sink, so the sink knows the same value as the sensor in case the sensor does not report. A sensor reports if the real reading deviates from the cached values by more than $\epsilon$.

3. Periodical approximate caching (P). This model is similar to approximate caching; however, every sensor caches the last reported readings for a set of episodes and uses the previous episode to determine its value. For example, for a 24-hour cycle, the value is checked against the reading from the same hour in the previous day. The lab data uses a 24-hour period, and the Asian temperature data uses a 12-month period.
TABLE 1

Abbreviations of Comparison Schemes

\begin{tabular}{llll}
\hline T & :TinyDB & $\mathbf{G}$ & :Global Average \\
$\mathbf{A}$ & :Approximate Caching & $\mathbf{P}$ & :Periodical Appr. Caching \\
$\mathbf{O}$ & :Ordinary Kriging & $\mathbf{O t}$ & :Temporal Ordinary Kriging \\
$\mathbf{S}$ & :Simple Average & $\mathbf{S t}$ & :Temporal Simple Average \\
$\mathbf{I}$ & :Inverse Distance & $\mathbf{I t}$ & :Temporal Inverse Distance \\
$\mathbf{K}$ & :Ken Model & & \\
\hline
\end{tabular}

- The state-of-the-art Ken model (K) [5]. This model refers to the Ken model with disjoint clique of size 5 . This technique has fixed-size static groups that send values to a leader that finds the optimal number to report from that group using a temporal model. Please refer to [5] for the details of the model. We group sensors according to spatial proximity instead of optimization using data reduction factors since spatial autocorrelation takes care of the majority of the problem.

- Ordinary kriging (O). This model uses E2K with ordinary kriging as the interpolation method. For the Asian temperature data, we removed the trend of the data for each sensor by subtracting periodic means obtained from the training data for each location. Each sensor will subtract that mean and use E2K for reporting its residual. The sink knows the same mean and will add it back upon receiving or interpolating the residual.

- Two schemes using the E2K framework but with simple interpolation methods without considering spatial structure:

1. Simple average (S). This scheme uses E2K with simple average as the interpolation method.

2. Inverse distance (I). This scheme uses E2K with inverse distance as the interpolation method.

For methods under the E2K framework, i.e., O, S, and I, their corresponding temporal versions are obtained by using a temporal model with $\mathrm{E} 2 \mathrm{~K}$ under the appropriate interpolation method and are called $\mathbf{O t}, \mathbf{S t}$, and It, respectively. The abbreviations are summarized in Table 1 for the convenience of the readers.

For all schemes, we implemented a program using Java to simulate the reporting behavior of each sensor node using the readings of each sensor and its neighboring sensors for a given time from the lab and Asia data sets. Unless otherwise specified, the radio range is $30 \mathrm{~m}$ and the error threshold is $0.5^{\circ} \mathrm{C}$ for the lab data, and the radio range is 5 degrees with an error threshold of $1{ }^{\circ} \mathrm{C}$ for the Asian temperature data.

For the lab data set the total messaging cost was estimated using a fixed multiple of the number of reporting sensors, i.e., we assumed that each sensor had to send a message to the central site using some fixed number of hops. This was done because the area and the number of sensors are too small for there to be a large number of hops. For the Asia data set, the central site was chosen to be the center of the map, and the number of hops to send a message was estimated taking into account the distance of each reporting sensor to the sink. For the lab data the first 


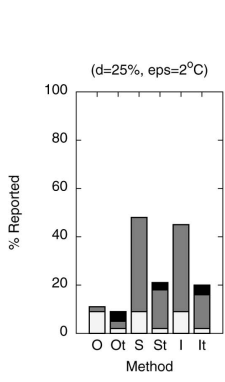

(a)

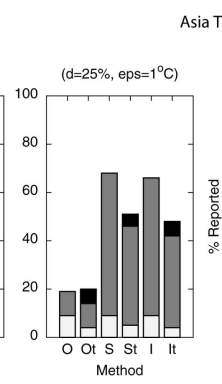

(b)

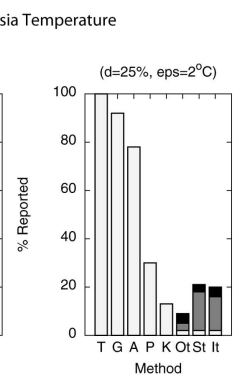

(c)

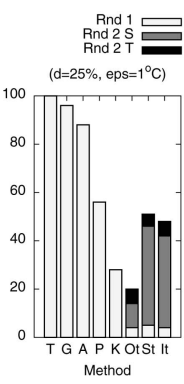

(d)
Fig. 6. Percentage report for Asia temperature data.

94 hours was used for training, and the next 452 hours was used for testing. For the Asian temperature data, the first 10 years was used for training, and the next 40 years was used for testing. We performed extensive experiments using various values for the parameters, and due to the limitation of space, we present a representative set.

\subsubsection{Performance on the Asia Temperature Data Set}

We first examine the percentage of sensors that actually reported for all the schemes. Note that the coordination overhead incurred when deciding which sensors to report and the communication cost needed to route the data to the sink are not factored in yet.

Figs. $6 \mathrm{a}$ and $6 \mathrm{~b}$ show that incorporating temporal schemes that allow E2K to dynamically utilize spatial and/or temporal autocorrelation depending on which is stronger in a given region does improve the performance for almost all schemes under E2K. For example, with $d=25$ percent and $\epsilon=2, \mathbf{S}$ has 9 percent of sensors to report in the first round and 39 percent of sensors to report due to spatial interpolation error (Rnd $2 \mathrm{~S}$ ) in the second round while the corresponding number for St are 2 percent and 16 percent. It has a small additional 3 percent of sensors to report due to temporal predication error in the second round (Rnd $2 \mathrm{~T}$ ). We can also see that the incorporation of a temporal scheme is particularly useful for the simple average and inverse distance schemes. The reason may be that simple average and inverse distance schemes do not use spatial autocorrelation and the spatial interpolation is less effective. Thus, the temporal scheme helps to improve the performance.

Figs. $6 \mathrm{c}$ and $6 \mathrm{~d}$ show that Ken and E2K with a temporal model outperform all the other schemes in terms of percentage of sensors that need to report. E2K using

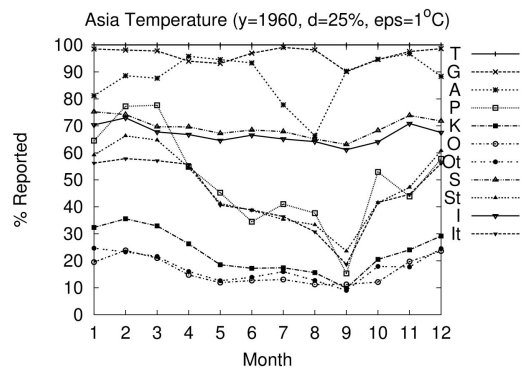

Fig. 7. Performance variations by month.

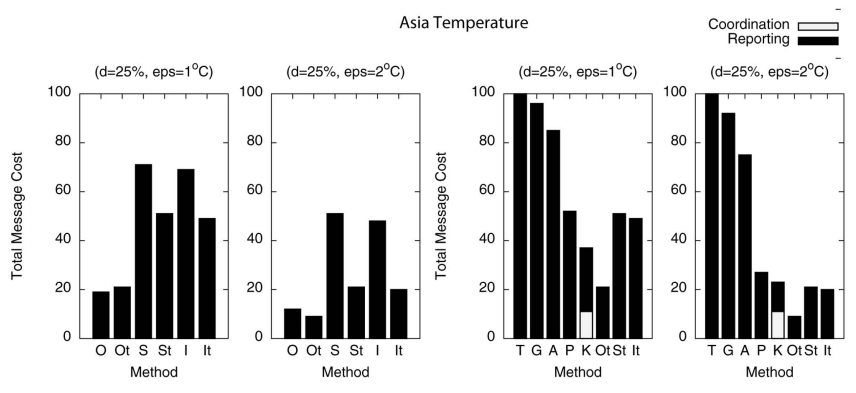

Fig. 8. Total messaging cost for Asia temperature data.

ordinary kriging reduces the percentage of sensors that need to report to 19 percent with $\epsilon=1$ and 12 percent with $\epsilon=2$ compared with 28 percent and 13 percent, respectively, by Ken.

To show the performance with respect to seasonal variation, we plotted a representative percentage of sensors that report for all the schemes for each month in the year 1960 in Fig. 7. In general, savings are more in the summer than during the winter, probably due to the smaller variation of the temperature in summer.

We now report the performance of the schemes with routing and coordination communication cost taken into consideration. All simple schemes and variations of E2K do not have coordination overhead if the interpolation neighborhood is within radio range. In Ken, group members need to send their readings to the group leader, resulting in coordination costs. Coordination cost is significant for a dense sensor network or high threshold value and ranges from 25 percent to 50 percent for Ken for the Asian temperature data set with 25 percent density. For this reason, Ot requires half of the total messages of Ken, as can be seen in Fig. 8.

\subsubsection{Performance on the Lab Data Set}

Similar to the results on the Asia temperature data set, temporal enhancement of schemes using the E2K framework does reduce the number of sensors that need to report substantially, e.g., 10-30 percent, as shown in Figs. 9a and $9 \mathrm{~b}$. When coordination and routing costs are not taken into consideration, Ken and the temporal E2K schemes outperform all other simple schemes. Ken requires 45 percent of the sensors to report for $\epsilon=0.5$ degree and 35 percent of the sensors to report for $\epsilon=1$ degree while the same numbers

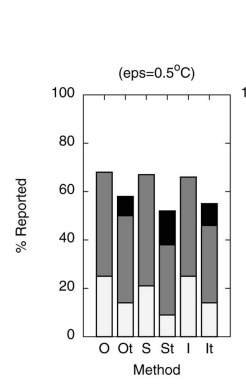

(a)

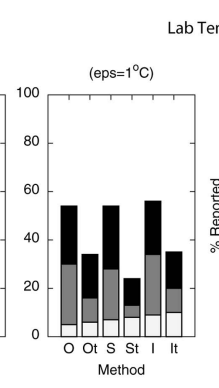

(b)

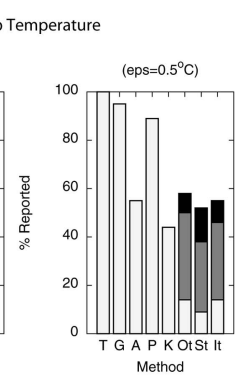

(c)

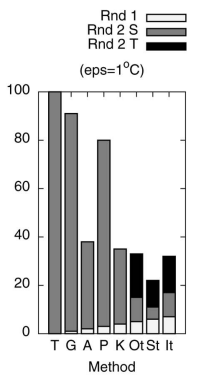

(d)

Fig. 9. Percentage report for lab data. 


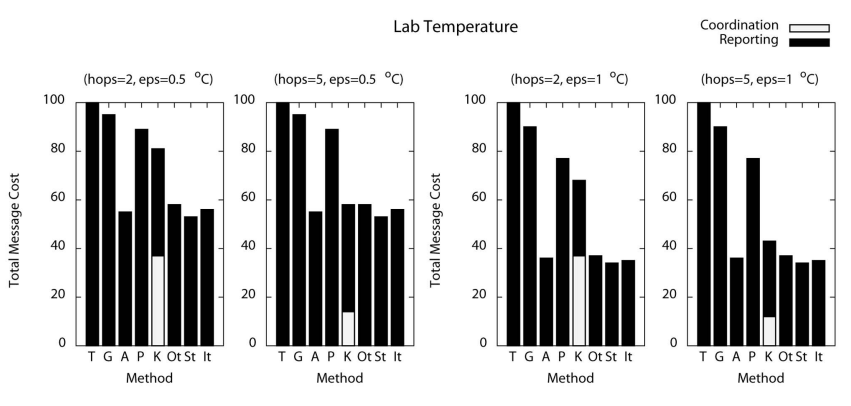

Fig. 10. Total messaging cost for lab data.

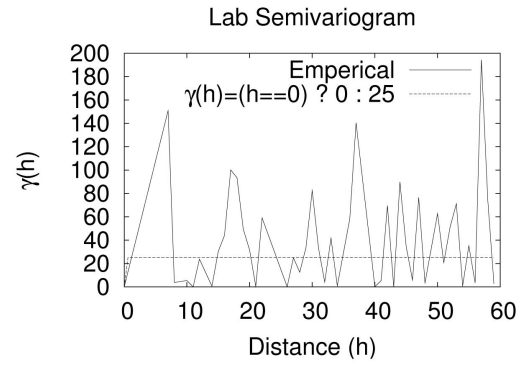

Fig. 11. Lab data empirical and theoretical variograms.

for Ot are 56 percent and 32 percent, respectively. In terms of total messaging costs, $\mathbf{O t}$ is slightly better than Ken for a large communication distance (five hops) to the sink and outperforms Ken by 26 percent to 35 percent of the cost of TinyDB for a small communication distance (two hops). Because the lab data set is relatively small, if the sink is one of the sensors, then the routing cost is also small. This makes coordination cost significant. As shown in Fig. 10, when the average number of hops from the sensor to the sink is two, the coordination cost for Ken is 37 percent, and the reporting cost is 44 percent, giving 81 percent total messaging cost. The coordination cost is almost half of the total messaging cost. When the distance to the sink is five hops, Ken improves significantly.

An interesting observation is that all schemes using the E2K framework, i.e., Ot, St, and It, achieve comparable results. The reason may be that the lab data is not from a typical outdoor environment and the spatial autocorrelation is not very strong. When using a pure nugget theoretical variogram, as shown in Fig. 11, kriging interpolation degenerates into a simple average model.

\subsubsection{Resilience to Sensor Network Failures}

When a sensor does not report, all schemes assume an interpolated value for that sensor, pretending that it is working and decides not to report. Let $n$ be the total number of sensors in the sensor field and $n_{\text {functional }}$ denote the number of functional sensors of each epoch. For all the functional sensors, Lemma 3 states that their readings are either exact readings (reporting sensors) or within $\epsilon$ (nonreporting sensors) for E2K schemes. For Ken, a group leader is preselected through an optimization scheme using training data. When a leader fails, all the readings that need to be reported inside the group will not be communicated to the sink and will be interpolated. Thus, a functional sensor with a failed leader may lead to an interpolated reading out of threshold in the sink. We denote the number of this type of sensors as $n_{\text {functional }}^{>\epsilon}$.

We plotted the real sensor failures including the percentage of all sensor failures and the percentage of leader sensor failures among the 54 sensors of the lab data sets in Fig. 12a in each hour. A sensor failure for an hour means that the sensor is never active for the whole hour. The total percentage of all sensor failures ranges from 5 percent to 100 percent (the whole sensor network died). The percentage of leader sensor failures ranges from 0 percent to 18 percent.

In Fig. 12b, we plotted the percentage of functional sensors reported within the threshold of 0.5 degree, i.e., $1-\frac{n_{\text {functional }}^{>\epsilon}}{n_{\text {functional }}}$, for the lab data. For E2K, this number is always 100 percent since all functional sensors are error bounded. For Ken, this number ranges from 67 percent to 100 percent (we ignored the period when the whole network died). So E2K allows better error control among normal functional sensors.

Since the Asia temperature data set does not contain real failures, we injected sensor failures in the Asia temperature data set by randomly selecting 5 percent to 25 percent of sensors to fail. Fig. 12c shows the percentage of functional sensors that reported within the threshold for both E2K and Ken with respect to the percentage of injected failures. Again, this number is 100 percent for E2K and decreases from 99 percent to 95 percent for Ken when the overall failure rate for each month is increased from 5 percent to 25 percent.

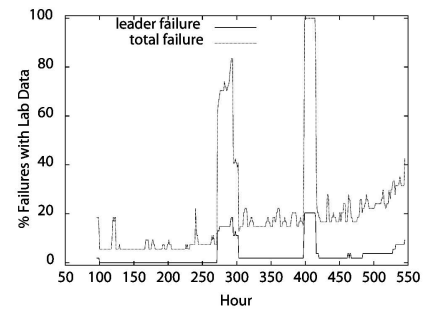

(a)

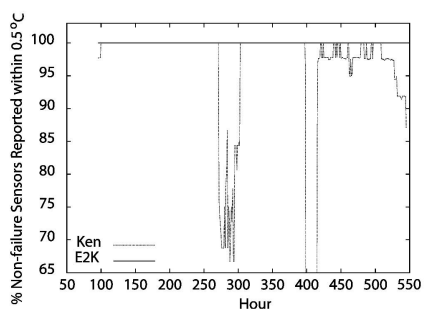

(b)

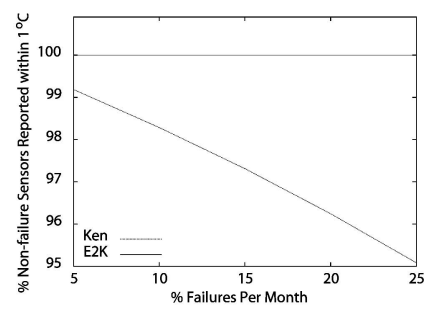

(c)

Fig. 12. Failures in lab data and Asia temperature data. (a) Failures in lab data. (b) Percentage within $\in$ in lab data. (c) Percentage within $\in$ in Asia temperature data. 


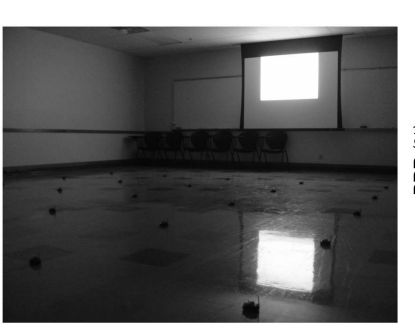

(a)

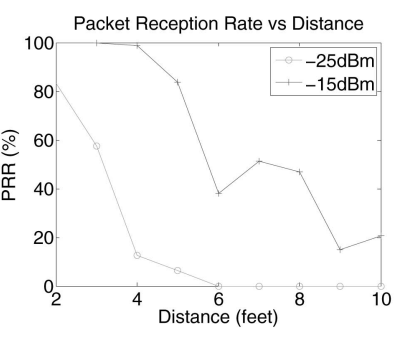

(b)
Fig. 13. (a) Real sensor network test environment. (b) Packet reception rate.

\subsection{Real Sensor Network Experiments}

We implemented the Naive algorithm (TinyDB), the E2K algorithm (with ordinary kriging), the Temporal algorithm (approximate caching), and the Temporal E2K (with ordinary kriging) on a network formed by 25 MICAz motes [15] as described at the beginning of Section 5, and the setup is as shown in Fig. 13a.

The sensors form a multihop routing tree rooted at the gateway sensor and periodically measure the phenomenon and report their readings to the gateway along the tree. The routing algorithm used in our framework is shortest path [37]. The routing protocol also provides reliable unicast communication by retransmitting packets that are not acknowledged by the destined sensors up to five times. However, for the first-round reporting, eavesdropping from neighboring sensors is not guaranteed since the neighbors are not intended neighbors. To enable data consistency and sensor coordination due to the unique iterative reporting, we also implemented a time synchronization protocol so that a networkwise clock is maintained. The time-sync protocol follows the Flooding Time Synchronization Protocol (FTSP) proposed in [28]. The pairwise FTSP features MAC-layer time stamping and clock drift compensation using linear regression, providing micro-second-level accuracy.

The onboard ADC translates a light intensity raw reading into an unsigned 10-bit integer. The calibration error among different sensors is 5 percent while the calibration error of the same sensor is about 0.2 percent. We chose to use the calibration error as the error threshold for the experiments. The relative distance $r$ is set to $\sqrt{2} \times 4 \mathrm{ft}$, that is, each node except the boundary nodes will have eight neighbors.

To evaluate the performance under a multihop network, we adjust the transmission power to a level where the communication range between two sensors will exceed $r$ while a multihop routing tree with a depth of more than 1 can be formed. Fig. $13 \mathrm{~b}$ depicts how the packet reception rate varies when a sensor reports a reading to its eight neighbors with different grid sizes in two transmission power setups. In our experiment, we choose $-15 \mathrm{dBm}$ so that more than 98 percent of the first-round data is successfully received. The observed maximum depth of the routing tree is three, and the average number of hops is 1.7.

We used the projector to vary the light intensity so that it would conform to a sine wave. Each sensor was configured to make 30 readings before stopping. By coordinating the
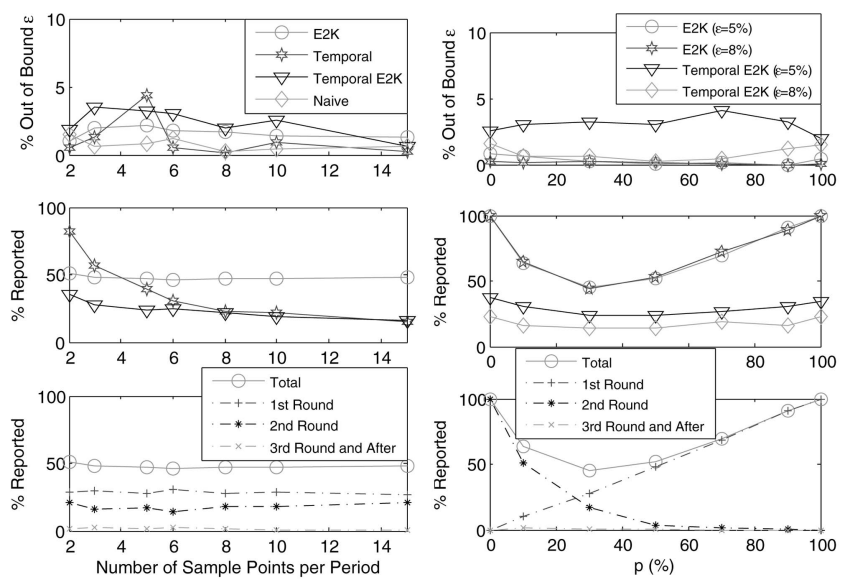

(a)

(b)

Fig. 14. (a) All with $p=30$ percent and $\epsilon=5$ percent. (b) All with a sampling rate of 5 samples per period and $\epsilon=5$ percent. (a) With respect to the sampling rate. (b) With respect to the probability of reporting.

projector with the sensors, the environment is reproducible so that different methods could be compared.

We stated error bounding for functional sensors in Lemma 3 assuming no packet loss. However, package loss is very common in real sensor networks. The top figure in Fig. 14a depicts the out-of-bound error rate due to package loss as the sample rate increases. As observed from the figure, all of the methods behave quite well with an error rate below 5 percent. The error rate of the naive method is actually the end-to-end packet loss rate. The Temporal E2K has the highest out-of-bound error rate because it transmits the fewest packets, and therefore, the information in each packet is more concentrated, so missing a packet will lead to high errors.

The middle figure in Fig. 14a examines the percentage of message hops compared with the naive method when sensors sample at each frequency. Note that the messages include all the initial transmission, retransmission, and relay. When sampling the light intensity at the peak and nadir points of the sine curve, the Temporal should have similar performance to the naive method, i.e., reporting 100 percent. However, Temporal still saves about 20 percent because the variances for sensors that were far away from the light source never exceeded the bound. As the number of sample points on the sine curve increases, the performance of Temporal also increases since there is a smoother transition, i.e., more temporal correlation, between adjacent readings. The E2K method saved nearly 50 percent by exploiting spatial correlation. As expected, Temporal E2K had the best performance with savings of more than 60 percent. The packet savings of E2K remains constant as the sample frequency increases. The performance of Temporal and Temporal E2K converge as temporal correlation becomes dominating.

The top figure in Fig. 14b shows the out-of-bound error versus $p$. From the figure, we can conclude that there is no correlation between the out-of-bound error rate and $p$.

The middle figure in Fig. 14b provides a guideline for how to choose the first-round reporting probability, $p$. Generally, $p=30$ percent saves the most. When we 


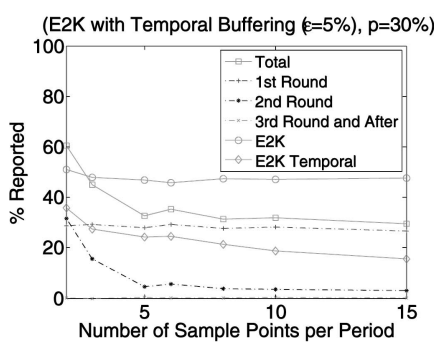

(a)

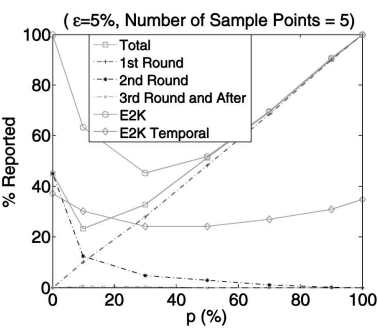

(b)
Fig. 15. (a) Experimental temporal E2K reporting versus sample rate. (b) Experimental temporal E2K versus probability to report.

increase the error bound from 5 percent to 8 percent, the E2K does not benefit while Temporal E2K saves an extra 10 percent. The discrepancy between the spatial calibration error ( 5 percent) and temporal calibration error ( 0.2 percent) should take the responsibility because the increased 3 percent error allowance has greater impact on the latter than the former.

The two figures at the bottom of Figs. $14 \mathrm{a}$ and $14 \mathrm{~b}$ decompose the percentages to report for different rounds of the E2K curves above them in Figs. 14a and 14b. The percentages to report for the third round and after remain almost constant and are very close to zero in all situations. In fact, there are no data after the third round at all from our observation. The third-round-reporting sensor readings actually represent the "discontinuity" of the map if interpolating data using both of the first and second round readings for nonreporting sensors and using actual readings for all reporting sensors. Because the third-round reporting rate is very small, the base station can interpolate for nonreporting sensors using both first- and second-round data without introducing too much "discontinuity."

\section{Conclusion AND Discussion}

In this work, we proposed the E2K framework, which works for any spatial interpolation method in general. When the spatial interpolation method works well, our framework is likely to save more on messaging cost.

The Temporal E2K algorithm selects pointwise between spatial and temporal interpolation. This approach would result in a "punctured" and noncontinuous final interpolated field. One possible solution is to perform a uniform spatiotemporal interpolation using sensor readings from spatiotemporal neighborhoods when a sensor tries to decide whether to report in place of the pointwise selection between spatial and temporal interpolation. However, a spatiotemporal variogram is needed for such spatiotemporal interpolation. Currently, we are not aware of any generally accepted spatiotemporal variograms. Thus, we implemented an experimental temporal method where the interpolation of a sensor reading $e_{0}$ is done by a simple averaging of sensor readings from $e_{0}$ 's spatiotemporal neighbors (we set the temporal neighbor as the previous epoch).

Fig. 15a compares the new temporal algorithm with E2K and E2K Temporal. Initially, when the number of sample points is two, the new algorithm performs the worst. That is because the old readings, for example, sampled at the peak, jeopardize the effectiveness of the interpolation process if they are used in predicting the readings at nadir points. As sample frequency increases, the new algorithm outperforms the spatial E2K and converges to the 30 percent line, the lower bound of the reported rate. The first-round transmission probability seems to play a big role when the sample frequency is high. Fig. 15b reveals the correlation between the report rate and the first-round transmitting probability. The figure clearly shows that the optimum point is at $\mathrm{p}=10$ percent, much less than 30 percent. This is not a surprise because the previous and current readings still provide 20 percent coverage of the field when interpolating data. After this point, the first-round transmission dominates the total transmission, and the performance converges to the E2K algorithm.

\section{ACKNOWLEDGMENTS}

This work was partially supported by the Texas Advanced Research Program under Grant 003594-0010-2006 and by the US National Science Foundation under Grants OCI-0636421 and CNS-0709285.

\section{REFERENCES}

[1] University of Delaware Surface Air Temperature Data, http:// climate.geog.udel.edu/ climate, 2008.

[2] M.H. Ali, W.G. Aref, and C. Nita-Rotaru, "SPASS: Scalable and Energy-Efficient Data Acquisition in Sensor Databases," Proc. Fourth ACM Int'l Workshop Data Eng. for Wireless and Mobile Access (MobiDE'05), 2005.

[3] B.A. Bash, J.W. Byers, and J. Considine, "Approximately Uniform Random Sampling in Sensor Networks," Proc. Int'l Workshop Data Management for Sensor Networks (DMSN '04), 2004.

[4] P. Bonnet, J.E. Gehrke, and P. Seshadri, "Towards Sensor Database Systems," Proc. Second Int'l Conf. Mobile Data Management (MDM '01), 2001.

[5] D. Chu, A. Deshpande, J. Hellerstein, and W. Hong, "Approximate Data Collection in Sensor Networks Using Probabilistic Models," Proc. 22nd Int'l Conf. Data Eng. (ICDE '06), 2006.

[6] J. Considine, F. Li, G. Kollios, and J. Byers, "Approximate Aggregation Techniques for Sensor Databases," Proc. 20th Int'l Conf. Data Eng. (ICDE '04), 2004.

[7] N.A.C. Cressie, Statistics for Spatial Data. John Wiley \& Sons, 1991.

[8] A. Deligiannakis, Y. Kotidis, and N. Roussopoulos, "Compressing Historical Information in Sensor Networks," Proc. ACM SIGMOD, pp. 527-538, 2004.

[9] A. Deshpande, C. Guestrin, S.R. Madden, J.M. Hellerstein, and W. Hong, "Model-Driven Data Acquisition in Sensor Networks," Proc. 30th Int'l Conf. Very Large Data Bases (VLDB '04), pp. 588-599, 2004.

[10] F. Emekci, S.E. Tuna, D. Agrawal, and E. Abbadi, "Binocular: A System Monitoring Framework," Proc. Int'l Workshop Data Management for Sensor Networks (DMSN '04), Aug. 2004.

[11] Q. Fang, F. Zhao, and L. Guibas, "Counting Targets: Building and Managing Aggregates in Wireless Sensor Networks," Technical Report P2002-10298, Palo Alto Research Center, 2002.

[12] D. Ganesan, B. Greenstein, D. Perelyubskiy, D. Estrin, and J. Heidemann, "An Evaluation of Multi-Resolution Storage for Sensor Networks," Proc. First Int'l Conf. Embedded Networked Sensor Systems (SenSys '03), 2003.

[13] S. Goel, A. Passarella, and T. Imielinski, "Using Buddies to Live Longer in a Boring World," Technical Report DCS-TR-558, Dept. of Computer Science, Rutgers Univ., 2004.

[14] B. Harrington and Y. Huang, "In-Network Surface Simplification for Sensor Fields," Proc. 13th ACM Int'l Symp. Advances in Geographic Information Systems (GIS '05), 2005.

[15] Crossbow Technology Inc., Intel Lab Data, http://www.xbow. com/Products/products.htm, 2008. 
[16] C. Intanagonwiwat, R. Govindan, and D. Estrin, "Directed Diffusion: A Scalable and Robust Communication Paradigm for Sensor Networks," Proc. ACM MobiCom, pp. 56-67, 2000.

[17] A. Jain, E.Y. Chang, and Y.-F. Wang, "Adaptive Stream Resource Management Using Kalman Filters," Proc. ACM SIGMOD, 2004.

[18] B. Karp and H.T. Kung, "GPSR: Greedy Perimeter Stateless Routing for Wireless Networks," Proc. ACM MobiCom, 2000.

[19] Y. Kotidis, "Snapshot Queries: Towards Data-Centric Sensor Networks," Proc. 21st Int'l Conf. Data Eng. (ICDE '05), pp. 131-142, 2005.

[20] B. Krishnamachari, D. Estrin, and S.B. Wicker, "The Impact of Data Aggregation in Wireless Sensor Networks," Proc. 22nd Int'l Conf. Distributed Computing Systems (ICDCS '02), pp. 575-578, 2002.

[21] D.R. Legates and C.J. Willmott, "Mean Seasonal and Spatial Variability in Global Surface Air Temperature," Theoretical and Applied Climatology, pp. 11-21, 1990.

[22] M. Li, D. Ganesan, and P. Shenoy, "PRESTO: Feedback-Driven Data Management in Sensor Networks," Proc. Third ACM/Usenix Symp. Networked Systems Design and Implementation (NSDI '06), May 2006.

[23] X. Li, Y. Jin Kim, R. Govindan, and W. Hong, "Multi-Dimensional Range Queries in Sensor Networks," Proc. First Int'l Conf. Embedded Networked Sensor Systems (SenSys '03), 2003.

[24] S. Madden, Intel Lab Data, http://berkeley.intel-research.net/ labdata/, 2008.

[25] S.R. Madden, M.J. Franklin, J.M. Hellerstein, and W. Hong, "TAG: A Tiny Aggregation Service for Ad-Hoc Sensor Networks," Proc. Fifth Symp. Operating System Design and Implementation (OSDI '02), 2002.

[26] S.R. Madden, M.J. Franklin, J.M. Hellerstein, and W. Hong, "Design of an Acquisitional Query Processor for Sensor Networks," Proc. ACM SIGMOD, 2003

[27] S.R. Madden, R. Szewczyk, M.J. Franklin, and D. Culler, "Supporting Aggregate Queries over Ad-Hoc Wireless Sensor Networks," Proc. Fourth IEEE Workshop Mobile Computing and Systems Applications (WMCSA '02), 2002.

[28] M. Maroti, B. Kusy, G. Simon, and A. Ledeczi, "The Flooding Time Synchronization Protocol," Proc. Second Int'l Conf. Embedded Networked Sensor Systems (SenSys '04), pp. 39-49, 2004.

[29] C. Olston, B. Thau Loo, and J. Widom, "Adaptive Precision Setting for Cached Approximate Values," Proc. ACM SIGMOD, 2001.

[30] S. Sandeep Pradhan and K. Ramchandran, "Distributed Source Coding Using Syndromes (DISCUS): Design and Construction," Proc. Data Compression Conf. (DCC'99), 1999.

[31] A. Scaglione and S.D. Servetto, "On the Interdependence of Routing and Data Compression in Multi-Hop Sensor Networks," Proc. ACM MobiCom, pp. 140-147, 2002.

[32] M. Sharifzadeh and C. Shahabi, "Supporting Spatial Aggregation in Sensor Network Databases," Proc. 12th Ann. ACM Int'l Workshop Geographic Information Systems (GIS '04), 2004.

[33] N. Trigoni, Y. Yao, A. Demers, J. Gehrke, and R. Rajaraman, "WaveScheduling: Energy-Efficient Data Dissemination for Sensor Networks," Internet draft, 2004.

[34] M.C. Vuran, B. Akan, and I.F. Akyildiz, "Spatio-Temporal Correlation: Theory and Applications for Wireless Sensor Networks," Computer Networks, vol. 45, no. 3, pp. 245-259, 2004

[35] M.C. Vuran and I.F. Akyildiz, "Spatial Correlation-Based Collaborative Medium Access Control in Wireless Sensor Networks," IEEE/ACM Trans. Networking, 2004

[36] H. Wackernagel, Mulitvariate Geostatistics. Springer, 1995

[37] A. Woo, T. Tong, and D. Culler, "Taming the Underlying Challenges of Reliable Multihop Routing in Sensor Networks," Proc. First Int'l Conf. Embedded Networked Sensor Systems (SenSys '03), pp. 14-27, 2003.

[38] Y. Yao and J. Gehrke, "The Cougar Approach to in-Network Query Processing in Sensor Networks," Proc. ACM SIGMOD, 2002.

[39] Y. Yu, R. Govindan, and D. Estrin, "Geographical and Energy Aware Routing: A Recursive Data Dissemination Protocol for Wireless Sensor Networks," Technical Report UCLA/CSD-TR-010023, Computer Science Dept., Univ. of California, Los Angeles, 2001.

[40] F. Zhao and L. Guibas, "Wireless Sensor Networks: An Information Processing Approach," Morgan Kaufmann Series in Networking, 2004.

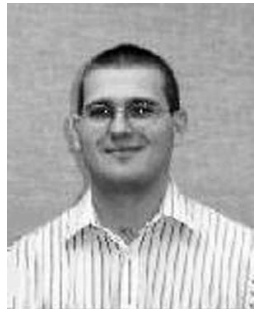

Brian Harrington received the BS, MS, and $\mathrm{PhD}$ degrees in computer science from the University of North Texas in 2002, 2003, and 2006, respectively. He is currently a software engineer at Yahoo!, working on grid-based content systems to support search.

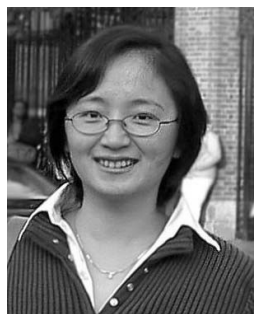

Yan Huang received the BS degree in computer science from Beijing University in July 1997 and the $\mathrm{PhD}$ degree in computer science from the University of Minnesota, Twin Cities, in July 2003. She is currently an assistant professor in the Department of Computer Science and Engineering, University of North Texas, Denton. Her research interests include geosensor networks, spatial databases, and data mining. Her research is supported by the Texas Advanced Research Program (ARP), Oak Ridge National Laboratory, and the US National Science Foundation. She is a member of the IEEE, the IEEE Computer Society, the ACM, ACM SIGMOD, and ACM SIGSPATIAL.

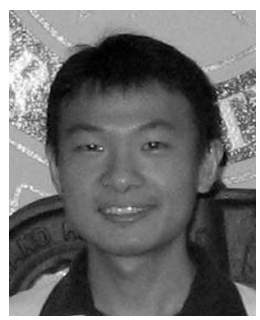

Jue Yang received the BS degree in computer science from Fudan University, Beijing, China, in 2005. He is currently a PhD candidate in the Department of Computer Science and Engineering, University of North Texas, Denton. His current research interests include statistical signal processing and wireless sensor networks. $\mathrm{He}$ is a student member of the IEEE.

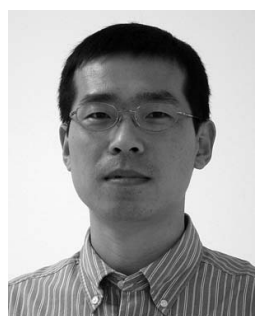

Xinrong $\mathbf{L i}$ received $\mathrm{BE}$ degree in electrical engineering from the University of Science and Technology of China, Hefei, in 1995, the ME degree in electrical engineering from the $\mathrm{Na}$ tional University of Singapore in 1999, and the $\mathrm{PhD}$ degree in electrical engineering from the Worcester Polytechnic Institute (WPI), Massachusetts, in 2003. From 2003 to 2004, he was a postdoc research fellow in the Center for Wireless Information Network Studies, WPI. Since 2004, he has been with the Department of Electrical Engineering, University of North Texas, Denton, as an assistant professor. His recent research has been focused on statistical signal processing for geolocation, wireless communications, and sensor networks. He is a member of the IEEE.

$\triangleright$ For more information on this or any other computing topic, please visit our Digital Library at www.computer.org/publications/dlib. 